\title{
Le traitement des "identifications diachroniques" dans des dictionnaires scolaires français : évaluation de pratiques et proposition de principes de rédaction alternatifs ${ }^{1}$
}

\author{
Nathalie Gasiglia \\ Université Charles de Gaulle - Lille 3 \\ UMR 8163 "Savoirs, Textes, Langage" \\ nathalie.gasiglia@univ-lille3.fr
}

Les dictionnaires scolaires français actuels qui ont un programme étymologique relativement consistant, le Dictionnaire Hachette junior [DHJ et Le Robert Junior illustré [RJI $]^{2}$ pour le cycle 3 de l'enseignement primaire, le Dictionnaire scolaire Hachette [DSH] et le Larousse Super Major [LSM] pour la transition avec le collège, fournissent des informations historiques pour une sélection de mots qui peuvent être issus du fonds latin, empruntés, construits avec des constituants d'origine étrangère ou française ${ }^{3}$, issus de troncations, d'antonomases, etc. ${ }^{4}$ D'autres informations relatives à l'histoire de la langue française sont fournies dans le Larousse Super Major et le Dictionnaire Maxi débutants ${ }^{5}$ [DMD] via des hors-texte, inclus respectivement dans un cahier autonome pour le premier (« Passeport pour la francophonie », pp. 8-11) et dans un post-texte du second, qui associe à une synthèse historique (pp. 1064-1068 de la «Petite histoire du français ») deux tableaux morphologiques ( La formation des familles de mots » et "Quelques mots-racines grecs ou latins », pp. 1036-1041). Ces données afférentes à l' «identification diachronique » des mots (cf. Hausmann \& Wiegand 1989 : 341 et 343 ) ont pour objectif de sensibiliser les jeunes lecteurs à l'histoire d'unités lexicales particulières et plus largement à celle de la langue française.

Je me propose d'évaluer la qualité relative des informations fournies aux consultants ${ }^{6}$. Après avoir pointé l'ambiguïté d'un introducteur d'origine polyvalent très employé (« vient de », § 1), j'examinerai comment les quatre dictionnaires scolaires pris en compte expliquent les changements phonographiques ou les constructions morphologiques qui séparent les mots français de leurs étymons ( $\$ 2$ ), puis comment ils glosent ces derniers afin de sensibiliser aux évolutions sémantiques des items et aux filiations de sens perceptibles au sein des familles étymologiques $(\S 3)$. La dernière partie sera consacrée à l'évaluation de la pertinence des repères fournis pour situer chronologiquement l'histoire de chaque mot au sein de l'histoire de la langue $(\S 4)$. Aux paragraphes 2,3 et 4 , après avoir décrit les pratiques observées et signalé les usages qui me semblent les plus adéquats ou au contraire inadaptés d'un point de vue pédagogique, je formulerai des principes de rédaction (mis en œuvre en annexe) d'un dictionnaire historique pour les jeunes qui permettraient aux lecteurs de plus pleinement tirer profit des informations fournies.

\section{Un introducteur d'origine polyvalent et usuel : « vient de »}

Si un rédacteur doit être concis dans la rédaction d'une information étymologique, il pourra être tenté d'employer la formule «vient de », qui permet d'introduire explicitement des éléments d'information relatifs à l'origine du mot décrit, mais qui exprime, pour qui sait la lire, que ce ne sont que des éléments, qui ne constituent pas une étymologie complète et précise. Cette formulation est ainsi être employée pour introduire, seuls ou combinés, un étymon (en particulier quand le mot français n'a pas la même forme que lui), une glose, une langue d'origine ${ }^{7}$, etc. Ceci peut être motivé par des contraintes de nombre de caractères pesant sur le texte des rubriques historiques de dictionnaires scolaires.

\subsection{Non-distinction des modes d'intégration des mots dans le lexique français}

Les dictionnaires Hachette ${ }^{8}$ et le $L S M$ utilisent fréquemment la formulation «vient de » pour signifier qu'un mot appartenant à une autre langue a été intégré au lexique français. C'est le cas par exemple s.v. décapiter, fromage, nucléaire, ovale, patience ou serf, tous d'origine latine. ${ }^{9}$ Parmi ces mots, les con- 
sultants devraient cependant pouvoir repérer : (i) que certains proviennent du fonds latin, comme fromage ou serf (qui, selon le DHLF, sont attestés, pour le premier, vers 1135 sous sa forme actuelle et en 1180 sous la forme sans métathèse formage, et, pour le second, vers 980 sous la forme serv puis à la fin du $\mathrm{X}^{\mathrm{e}}$ siècle sous la forme serf) (ii) que d'autres, par contre, ont été empruntés au latin, comme patience (attesté en français vers 1120) ou décapiter (utilisé depuis le début du XIV siècle) et (iii) que d'autres encore ont été construits en français avec des constituants ou un sous-ensemble de leurs constituants qui viennent du latin comme nucléaire (sur nucleus, 1834) ou ovale (sur ovum, 1377).

\subsection{Non-distinction des processus menant des étymons aux mots français}

Les dictionnaires Hachette et le LSM utilisent fréquemment la formulation «vient de » pour signifier qu'un mot a été intégré au lexique français avec modification de la forme d'origine, sans que cette formulation permette d'identifier quelles évolutions phonographiques ou quels procédés de création d'unités lexicales se sont opérés, et dans quelle langue (langue source ou français). C'est le cas par exemple s.v. armistice, aube, bourse, décapiter, fromage, linoléum, nucléaire, ovale ou plaisir ${ }^{10}$, où l'usage de cet opérateur polyvalent ne permet pas de distinguer entre différents processus : évolution phonographique en français pour bourse (à partir du bas latin bursa, qui l'a emprunté au grec bursa); métathèse en français pour fromage à partir de formage ; conversion en latin (adjectif albus $\rightarrow$ nom alba) pour aube ("moment du jour" et "robe") ; conversion en français pour plaisir (verbe plaisir hérité du latin (placere) $\rightarrow$ nom), pour lequel les Hachette ne fournissent pas d'étymologie ; dérivation en latin pour décapiter (decapitare, de de-, -capit-, allomorphe de caput, et -are) ; dérivation en français pour nucléaire et ovale (base latine et suffixe français); composition en latin médiéval (armistitium, de arma et statio) pour armistice ${ }^{11}$; composition en anglais (linoleum) avec des constituants latins (linum et oleum) pour linoléum ${ }^{12}$.

\section{Décrire les évolutions de formes et les constructions de mots}

Même quand les descriptions des évolutions morphologiques sont présentes, elles sont souvent trop sommaires ou implicites dans les dictionnaires scolaires, ce qui est le reflet de sous-entendus fréquemment observables dans les composants étymologiques de dictionnaires généraux destinés aux adultes. Il semble cependant dommageable de ne pas développer plus didactiquement ces descriptions pour expliciter certaines règles ou sensibiliser à l'existence de mécanismes récurrents. Les exemples ci-dessous inventorient le manque de motivation des formes ou des changements donnés à voir. Ils ne couvrent pas tous les cas de figure observables mais permettent d'évoquer différentes inadéquations descriptives concernant soit des variations phonographiques de mots passés d'une langue à une autre, soit des constructions d'items dont certaines étapes sont omises bien qu'utiles à la compréhension du propos ou qui impliquent des constituants partagés avec d'autres mots français construits de manière comparable mais au sujet desquels, éventuellement, rien n'est signalé.

\subsection{Variations graphiques subies par les emprunts}

Les patrons de rédaction des Hachette sont prévus de manière telle que les rédacteurs mentionnent le mot dont ils fournissent l'histoire au sein du composant étymologique. Outre que cette information n'est pas indispensable puisqu'il s'agit de l'item mentionné en adresse et que le $L S M$, qui ne le répète pas, n'en est pas moins clair, la mention du mot français peut conduire à formuler des informations fausses. Les emprunts d'unités polylexicales à l'anglais, en particulier, sont volontiers orthographiés avec des traits d'union en français remplaçant des espaces ou un accolement des constituants dans la langue source, ce que ne reflètent pas des textes comme « Rocking-chair est un mot anglais » ou «Skate-board est un mot anglais $»^{13}$, qui devraient respectivement contenir rocking chair et skateboard ${ }^{14}$. La variation graphique qui est passée sous silence mériterait pourtant d'être valorisée pour montrer que le processus d'intégration de ces mots au lexique français peut s'accompagner de distorsions orthographiques. 


\subsection{Décomposition morpho-sémantique des formes empruntées}

Pour des formes empruntées comme agenda, les Hachette fournissent simplement une glose, le $L S M$ va à peine plus loin en évoquant la racine du verbe agir, et le $R J I-C E$ associe à ces deux informations le fait qu'il s'agit d'une forme du verbe agere (cf. [1] ci-dessous). Mais aucun n'explique que cette forme latine correspond à celle de l'adjectif verbal d'obligation, qui, certes, n'existe pas en français mais qui est reconnaissable à la présence du -nd-(-ndus) et que les élèves rencontrent, même s'ils n'en ont pas conscience, quand ils emploient par exemple les mots légende ("ce qui doit être lu"), dividende (littéralement "ce qui doit être divisé") et multiplicande ("ce qui doit être multiplié"), trois termes scolaires 15 , et offrande ("ce qui doit être offert"). Tous ces mots sont à la nomenclature des quatre dictionnaires étudiés, mais seul le LSM associe une étymologie à l'un d'eux (légende), et encore est-elle implicite sur ce point (« qui signifie «ce qui doit être lu»»). Aucun n'explique non plus que le - $a$ d'agenda est une marque flexionnelle de neutre pluriel que l'on retrouve dans visa, passé lui aussi au singulier en français, et dans desiderata, employé aux deux nombres avec une dominance du pluriel ${ }^{16}$. La description de cette marque est toutefois moins utile que celle de la précédente, puisque peu de mots en ont conservé la trace en français et que seul visa est sémantiquement comparable à agenda, étant lui aussi le produit d'une métonymie (choses à faire $\rightarrow$ registre où elles sont écrites ; choses vues $\rightarrow$ marque apposée sur celles qui ont été vues).

[1] s.v. agenda • «En latin, agenda signifie «les choses qu'il faut faire ».» (DHJ et $D S H) \bullet$ «Mot latin qui signifie « ce qui doit être fait» et dont on retrouve la racine dans agir.» $(L S M) \bullet$ «Ce mot latin veut dire «ce qui doit être fait, choses à faire ». C'est une forme du verbe agere signifiant «faire, s'occuper de», verbe qui est à l'origine du verbe agir. » (RJI-CE)

\section{3 Évolutions phonographiques régulières}

Certains étymons latins présentés ont une finale de masculin singulier en - us qui ne s'est pas conservée dans les mots français correspondants. C'est le cas par exemple s.v. nucléaire ou serf (cf. [2] et [3]), pour reprendre deux items déjà évoqués. Sans détailler les évolutions phonographiques de ces mots en français, il pourrait être bienvenu que les articles et/ou les paratextes des ouvrages y sensibilisent les jeunes consultants et leur fournissent quelques clés : en indiquant par exemple que la finale -us (et d'autres) a été tronquée quand le mot latin a été utilisé comme base de dérivation (nucle(us) + -aire) et qu'elle est sujette à avoir disparu dans les mots français hérités du latin (servus $\rightarrow \operatorname{serf})$.

[2] s.v. nucléaire • « Nucléaire vient du latin nucleus qui signifie «noyau ». » (DHJ et $D S H) \bullet$ «Vient du latin nucleus qui signifie «noyau ». » $(L S M) \bullet$ "Cet adjectif s'emploie en physique pour parler de ce qui concerne la partie située au cœur de l'atome, le noyau, autour duquel tournent les électrons. La découverte de l'existence du noyau de l'atome en 1911 a permis d'étudier l'énergie colossale que l'on peut en tirer lorsqu'on arrive à le casser ou à le fusionner avec un autre noyau. Le mot nucléaire vient de nucleus qui veut dire «noyau, pépin ». » $(R J I-C E)$

[3] s.v. serf • «Serf vient du latin servus qui signifie «esclave».» (DHJ et DSH) - «Vient du latin servus qui signifie « esclave».» $(L S M) \bullet$ « Ce mot vient du latin servus qui signifie «esclave». L'absence de liberté réunit l'esclave antique et le serf médiéval qui dépend du seigneur qui lui confie sa terre et lui donne sa protection. Les mots servir, asservir, servile et servitude ont la même origine. » (RJI-CE)

\subsection{Procédés de construction, constituants impliqués et allomorphes}

Pour les mots construits par dérivation (dans leur langue d'origine ou en français), les dictionnaires observés fournissent la base de cette dérivation (caput pour décapiter, nucleus pour nucléaire, cf. [4] et [2]), mais aucun ne mentionne les affixes impliqués, alors qu'ils peuvent se rencontrer dans d'autres mots dérivés et intégrés au français ou dérivés en français, et, quand il s'agit de dérivations latines, aucun ne fournit le dérivé. Il serait plus explicite d'indiquer que décapiter est la forme française du verbe decapitare, qui a été emprunté au latin où il avait été construit avec le préfixe privatif de-, qui a donné dé- en français, 
la base -capit- allomorphe de caput qui signifie "tête" et la finale verbale -are. Rien ne s'opposerait non plus à ce que soit explicité que nucléaire est construit en français avec le constituant latin nucle(us) qui signifie "noyau" et le suffixe -aire qui permet de construire des adjectifs de relation et que l'on retrouve dans urticaire (cf. [5]), de formation comparable, pour lequel le suffixe n'est pas signalé non plus.

[4] s.v. décapiter • «Décapiter vient du latin caput qui signifie « tête ». » (DHJ et $D S H) \bullet$ «Vient du latin caput qui signifie « tête » et que l'on retrouve dans capitaine, capital, capiteux. » $(L S M) \bullet$ «Ce verbe vient du latin qui l'a formé à partir du mot caput qui veut dire «tête ». Caput signifie aussi « celui qui est à la tête, personnage principal », ce qui explique que le mot capitaine est de même origine. » (RJI-CE)

[5] s.v. urticaire • «Urticaire vient du latin urtica qui signifie «ortie», car l'urticaire ressemble aux boutons occasionnés par les orties. » (DHJ et $D S H)$ - «Vient, comme ortie, du latin urtica. » (LSM)

\title{
2.5 Étapes de construction
}

Dans une large partie des composants étymologiques, les mots français qui motivent leur présence ne sont pas de même catégorie grammaticale que les étymons fournis et, quand il s'agit de dérivations complexes, les consultants n'ont aucun moyen de reconstruire validement les étapes qui ont précédé l'intégration du mot au français. Par exemple, s.v. patience (cf. [6]), les quatre dictionnaires s'accordent pour expliquer que le nom français vient du latin pati, glosé par «supporter» dans les Hachette et le $L S M$ et par «supporter, endurer, subir » dans le RJI-CE. Cette information, qui met la puce à l'oreille, un nom ne pouvant venir d'un mot qui a un sens verbal que par dérivation, dissimule en fait le parcours constructionnel de l'étymon latin, ce à quoi il pourrait utilement être remédié par l'indication que le nom français patience a été emprunté au latin patientia, lui-même dérivé dans cette langue de la base patiens, forme du participe présent du verbe pati, au moyen du suffixe -ia, qui permet de construire des noms de qualité.

\begin{abstract}
[6] s.v. patience • «Patience vient du latin pati qui signifie « supporter ». » (DHJ et $D S H) \bullet$ «Vient du latin pati qui signifie « supporter ». » $(L S M) \bullet \ll$ La patience est la qualité qui permet de supporter sans rien dire, sans se décourager, sans s'énerver les aspects désagréables d'autrui ou de l'existence. Le mot patience vient du latin, du verbe pati qui veut dire «supporter, endurer, subir » avec l'idée de résignation, de persévérance. Le verbe pâtir, les mots compassion et compatissant sont de même origine. $(\rightarrow$ sympathie) $)(R J I-C E)$
\end{abstract}

La même observation vaut quand ce n'est pas l'étymon latin mais sa traduction française qui est fournie. Par exemple, s.v. abréger dans le $L S M$ (cf. [7]), le verbe français est corrélé à une base adjectivale latine non exprimée, ce qui ne permet pas de déduire si la dérivation s'est faite en latin ou en français : si la mention de l'adjectif est jugée utile, il ne peut être remédié à cette indétermination qu'en expliquant que l'adjectif latin brevis, duquel est issu l'adjectif français bref, a servi de base au verbe dérivé bas latin abbreviare, qui a été hérité en français et a évolué jusqu'à avoir la forme abréger que nous connaissons.

$$
\text { [7] s.v. abréger • «Vient d'un mot latin qui signifie «bref ». » }(L S M)
$$

\subsection{Cheminement initial de mots empruntés au français par d'autres langues puis réempruntés par le français}

Pour certains mots que l'anglais avait empruntés au français ou calqués sur le français avant que le français n'emprunte à son tour les mots anglais résultants, certains composants étymologiques indiquent cet aller et retour. Si les quatre dictionnaires signalent que stress (cf. [8]) est un mot anglais, le RJI-CE est le seul à décrire son double cheminement et l'aphérèse survenue lors de l'emprunt par l'anglais. Pour gentleman (cf. [9]), le RJI-CE fournit les mêmes types d'informations alors que le LSM décompose l'item anglais (sans évoquer cependant l'emprunt au français de son patron de construction). Il peut y avoir débat pour déterminer si l'origine des mots anglais étymons de mots français doit ou non être détaillée dans une notice étymologique de dictionnaire scolaire français, mais il semble important que les consultants 
puissent comprendre ce qui motive les variations de sélection d'informations observables. Peut-être sontelles, dans le cas présent, indexées sur l'opposition emprunt / calque.

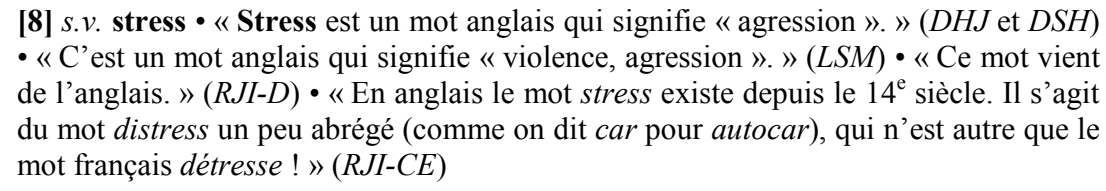

[9] s.v. gentleman - «Gentleman est un mot anglais qui signifie « gentilhomme ». » $(D H J) \bullet$ «Gentleman signifie « gentilhomme» en anglais. » $(D S H) \bullet ~ « C$ 'est un mot anglais, formé de gentle, «gentil », et de man, « homme ». » $(L S M) \bullet$ « Ce mot vient de l'anglais. » $(R J I-D) \bullet$ « Ce mot anglais est formé sur le modèle du français gentilhomme, à partir de l'adjectif gentle qui signifie «noble » et de man qui veut dire «homme». Le français distingue les deux mots : seul le gentilhomme fait partie de la noblesse ; le gentleman se remarque par ses bonnes manières et son excellente éducation. » $(R J I-C E)$

\subsection{Familles étymologiques}

Pour les étymons qui sont partagés par plusieurs mots français ${ }^{17}$, il est relativement usuel que tous les articles décrivant ces mots n'intègrent pas de composant étymologique et que l'un (ou un sous-ensemble) d'entre eux signale l'étymon partagé. C'est le cas par exemple s.v. ovale (cf. [10]), où les Hachette notent qu'ovaire et ovule partagent le même étymon, ce que le RJI-CE fait pour ovaire, ovipare et ovule, sans que les articles qui décrivent les items mentionnés offrent d'informations étymologiques. Le $L S M$ est plus complet $^{18}$ : il propose une étymologie pour ovaire et ovule, qui sont mentionnés s.v. ovale, et une pour ovipare, et il mentionne pour chacun l'étymon ovum. ${ }^{19}$ Il ne laisse cependant entrapercevoir la famille étymologique qu'à partir de ovale, ce qui pourrait être amélioré soit en systématisant les renvois ${ }^{20}$, soit en introduisant des hors-texte qui schématiseraient les liens étymologiques, à la manière de ce qui est présenté dans le $D H L F$ (cf. la « Table des schémas » (pp. 4301-4302)) et de ce qui est proposé en annexe 2.

[10] s.v. ovale $\bullet$ «Ovale vient du latin ovum qui signifie « œuf», et que l'on retrouve dans ovaire et dans ovule. » (DHJ et $D S H)$ - «Vient, comme ovaire, du latin ovum. » $(L S M) \cdot$ «Un visage ovale, un ballon ovale ont la forme d'une courbe fermée et allongée, la forme d'un œuf. L'adjectif ovale a pour origine le latin ovum qui veut dire « œuf ». Des mots concernant la reproduction comme ovaire, ovipare ou ovule ont la même origine. » $(R J I-C E)$

\subsection{Orientations conclusives pour la description des évolutions de formes et des constructions de mots}

À partir de cette sélection d'exemples, quelques principes rédactionnels semblent devoir être suggérés : le premier consiste à attirer l'attention sur les variations phonographiques observables diachroniquement et synchroniquement (entre deux langues ou au sein d'une seule), le second à bien expliquer quelles formes sont impliquées dans un emprunt ou une construction morphologique et à signaler les allomorphies identifiables $^{21}$, le troisième à nommer les mécanismes responsables des changements de forme présentés, le quatrième à permettre que, pour chaque mot figurant à la nomenclature, les informations étymologiques qui le concernent soient accessibles à partir de l'article qui le décrit.

À titre d'exemple, la description étymologique d'agenda (cf. annexe 1) pourrait être enrichie afin de pointer (a) les étapes de la construction du mot en bas latin, (b) celles de son entrée en français, (c) les liens étymologiques qu'il entretient avec d'autres mots français et (d) les éléments morphologiques repérables et partagés. Concernant la description des évolutions de formes et des constructions de mots, le patron de rédaction, qui est proposé en annexe, illustre certaines des orientations précédemment posées. Il permet en outre de constater que celles-ci ont des incidences sur la manière rédactionnelle : elles ont conduit à présenter l'histoire du mot de manière chronologique (du verbe latin agere au nom latin agenda 
emprunté en français), à établir un parallèle entre les constructions observées en latin et d'autres faites en français (deux conversions successives : verbe $\rightarrow$ adjectif et adjectif $\rightarrow$ nom) et à ébaucher un schéma de famille étymologique (cf. annexe 2). Une réécriture adaptée au support de consultation et associée à une présentation judicieusement conçue devrait s'employer à résoudre les problèmes de lisibilité encore patents en particulier en fournissant des aides à la pleine compréhension de ce qui est expliqué : définitions des termes, localisations géographiques et temporelles des langues et états de langues évoqués, etc.

\section{Sensibiliser aux évolutions et filiations de sens}

Il est d'usage, dans les descriptions étymologiques, de fournir des gloses qui doivent permettre aux consultants de bien comprendre les évolutions sémantiques des mots. Dans les dictionnaires destinés à de jeunes lecteurs, la rédaction de ces gloses nécessite une attention particulière puisqu'elle doit s'adapter aux compétences linguistiques du public cible : vocabulaire limité, maîtrise imparfaite de la polysémie des mots et ignorance des changements sémantiques diachroniques. Pour autant, simplifier ne doit pas amener à dénaturer les éléments transmis. L'échantillon d'exemples proposés ci-après permet d'évoquer quelques-uns des problèmes observés : glose unique réunissant celles de plusieurs composants d'un mot complexe, choix de sens contestable ou absence de repère chronologique quand des variations sémantiques sont attestées pour un étymon et qu'une seule glose est exploitée dans la description étymologique, ou encore sélection implicite et éventuellement injustifiée des informations étymologiques fournies, qui ne portent plus que sur un sous-ensemble des sens synchroniques décrits, sans que ce soit signalé.

\subsection{Précision des gloses}

C'est probablement dans un souci de simplification que les deux Hachette formulent une glose synthétique approximative dans certains composants étymologiques de mots complexes compositionnellement interprétables comme armistice (cf. [11]), pour lequel les deux autres dictionnaires étudiés ont préféré gloser chaque constituant, ce que le $R J I-C E$ a complété par une glose du mot construit.

[11] s.v. armistice • «Armistice est formé de deux mots latins qui signifient « les armes restent immobiles ». » $(D H J$ et $D S H) \cdot$ «Vient du latin arma qui signifie « armes » et sistere qui signifie « arrêter ».» $(L S M) \bullet$ « Ce mot vient du latin; il est composé à partir de arma qui signifie " arme » et du verbe stare qui veut dire "être immobile ». L'armistice est donc un « arrêt des armes », tout comme le solstice est un « arrêt du Soleil ». » $(R J I-C E)$

\subsection{Sélection de sens d'étymons polysémiques}

Si les gloses soutiennent la compréhension de l'histoire des mots, pour un étymon qui est polysémique (au moins durant une partie de sa durée d'usage) il semble utile de gloser le ou les sens le(s) plus directement impliqué(s) dans l'étymologie du mot français qui motive la description, surtout quand il(s) correspond(ent) au(x) sens moderne(s) du mot. Dans le cas du mot arène (cf. [12]), les quatre dictionnaires indiquent qu'il vient du latin arena, auquel ils associent la glose « sable », qu'ils justifient par le fait que le sol des aires centrales de cirques ou d'amphithéâtres romains antiques était sablé. Mais si ce sens d'arena perdura longtemps pour arène ${ }^{22}$ et fut aussi actif dans quelques mots français sortis de l'usage (aréneux, arénifère, arénuleux...), il ne survit guère aujourd'hui que dans arénicole, que ne répertorie que le $D S H$, sans d'ailleurs lui associer d'étymologie. Par contre, les deux emplois d'arène(s) décrits par les quatre dictionnaires (la piste circulaire sablée qui est au centre d'un amphithéâtre et, au pluriel, l'amphithéâtre lui-même) procèdent d'emplois déjà attestés pour l'étymon latin arena $^{23}$. La mention du seul sens de «sable » pour arena par les dictionnaires scolaires, si elle n'est pas fautive puisque ses différents autres sens viennent de ce sens initial, masque cependant que le mot latin a été polysémique et que le mot français, qui a progressivement intégré plusieurs de ses sens dérivés, a finalement perdu son sens premier.

[12] s.v. arène • «Arène vient du latin arena $^{24}$ qui veut dire « sable », car la piste est recouverte de sable. » $(D H J$ et $D S H) \bullet$ «Vient du latin arena, « sable ». La partie cen- 


\begin{abstract}
trale des amphithéâtres et des cirques romains était sablée. » $(L S M) \bullet$ « Ce mot vient du latin arena qui signifie «sable ». L'amphithéâtre antique comporte une muraille circulaire à arcades, des gradins pour les spectateurs, et une arène, qui est l'espace central recouvert de sable. Les jeux du cirque, les combats de gladiateurs ou d'animaux se déroulent dans l'arène. Aujourd'hui, on dit les arènes en parlant d'un amphithéâtre romain (les arènes de Nîmes, d'Arles). » (RJI-CE)
\end{abstract}

Un mot polysémique en synchronie peut par ailleurs n'avoir transmis que l'une ou l'autre de ses valeurs aux mots dont il est l'étymon. C'est au rédacteur de chaque étymologie qu'il revient de bien repérer chaque acception utile et, le cas échéant, de la dater. Cela n'est pas pleinement le cas, par exemple, dans le $R J I$, où la glose de l'étymon de shampooing diffère d'un article à l'autre (cf. [13]) : dans le RJI-CE elle exprime bien le sens de l'étymon repris par l'emprunt français ${ }^{25}$ alors que dans le $R J I-D$ elle est étymologique mais non pertinente (elle concerne le premier sens du verbe anglais to shampoo, qui s'est enrichi et a permis de créer par conversion un nom anglais polysémique ("massage" et "lavage") dont le français n'a retenu que le deuxième sens quand il l'a emprunté). Cette coprésence de données qui ne se recoupent pas ne documente pas clairement les lecteurs.

\begin{abstract}
[13] s.v. shampooing ${ }^{26} \bullet$ «On écrit aussi shampoing. En anglais le verbe to shampoo signifie « masser ». » $(R J I-D) \bullet$ « C'est un mot anglais qui vient du verbe to shampoo qui signifie « faire un shampooing », provenant d'un verbe indien qui veut dire « masser, presser ». » $(R J I-C E)$
\end{abstract}

\title{
3.3 Prise en compte de l'étymologie de différents sens d'items polysémiques
}

Dans les dictionnaires scolaires, les descriptions de sens attestés en synchronie pour un mot polysémique ne sont pas ordonnées en fonction de la chronologie relative de leur apparition. En conséquence, les informations étymologiques fournies ne portent pas nécessairement sur le premier sens décrit et c'est uniquement leur formulation qui permettra (ou pas) de définir leur portée. Les deux dictionnaires pour les collégiens ${ }^{27}$ ne fournissent d'étymologie de calcul (cf. [14]) que pour le(s) sens mathématique(s) de cet item (décrit(s) s.v. calcul 1 et 2 dans le $D S H$ et 1 calcul 1 dans le $L S M$ ), et pas pour son sens médical (décrit s.v. calcul 4 dans le $D S H$ et $\mathbf{2}$ calcul dans le $L S M$ ). Ce choix peut paraître étrange dans la mesure où c'est pour le sens médical que la relation est directe entre l'étymon calculus et calcul, sous la forme d'un emprunt daté de 1540. Par ailleurs, dans les étymologies fournies, le $D S H$ et le $L S M$ n'explicitent pas le cheminement indirect qui mène au sens mathématique de calcul, depuis le nom latin classique calculus via le verbe bas latin calculare, son emprunt par le français sous la forme calculer attestée en 1372 et la conversion nominale de celui-ci en calcul observée en 1484.

[14] s.v. calcul • «Calcul vient du latin calculus qui signifie « petit caillou » car on a compté d'abord avec des cailloux.» $(D S H) \bullet$ «Vient du latin calculus qui signifie « caillou » car, autrefois, on utilisait des cailloux pour compter. » (LSM, s.v. 1 calcul)

\subsection{Orientations conclusives pour la sensibilisation aux évolutions et filiations de sens}

Les différents exemples analysés ne couvrent pas l'ensemble des configurations possibles, mais ils fournissent le matériau utile pour asseoir trois directives rédactionnelles : la première consiste à bien sélectionner les éléments historiques les plus adéquats et les mieux explicables en fonction de l'état de langue actuel et supposé connu des consultants, la seconde, à bien veiller à indiquer sur quoi portent les gloses formulées et quand elles ont été valides, si elles ne l'étaient que pour des états de langue anciens, et la dernière, à créer des liens entre les éléments de la description étymologique et les subdivisions d'articles qui décrivent les sens différents coprésents synchroniquement.

Le mot acte, un des mots de la famille étymologique d'agenda présentés dans le schéma fourni dans l'annexe 2, n'a pas fait l'objet de descriptions étymologiques dans les quatre dictionnaires étudiés. Sa polysémie et l'histoire de chacun de ses emplois en font cependant un exemple intéressant. Pour présenter les sens de cet item, les Hachette établissent un article à trois subdivisions, le $L S M$ propose trois articles et 
le $R J I-D$ deux, le second étant subdivisé. Trois sens sont décrits ("document officiel", "chose faite" et "subdivision de pièce de théâtre"), distribués de manières différentes dans chaque ouvrage et ne reflétant les datations d'usage en français que dans le $R J I-D$, où ce n'est pas indiqué et donc pas perceptible. ${ }^{28} \mathrm{La}$ description étymologique devrait reprendre les trois parcours qui ont permis d'obtenir les trois sens du nom français acte (i) à partir d'un nom neutre construit en latin classique par dérivation du participe passé du verbe agere (actum "chose faite", emprunté au XVI ${ }^{\mathrm{e}}$ s. avec ce sens), dont la forme du pluriel, acta, s'était spécialisée dès le latin classique pour désigner les "pièces juridiques", premier sens emprunté (au $\mathrm{XIV}^{\mathrm{e}} \mathrm{s}$.), et (ii) d'un autre nom masculin, actus, dérivé lui aussi d'agere et qui a permis de nommer une "pièce de théâtre" puis une "subdivision principale de celle-ci", le dernier sens emprunté (au XVI s.). Ces parcours, qui figurent sur le schéma de l'annexe 2, devraient être textuellement présentés pour acte, soit en établissant trois descriptions étymologiques, une par sens décrit, soit en n'en créant qu'une qui intégrerait des renvois aux descriptions de chaque sens.

\section{Ancrer l'histoire des mots dans une chronologie accessible}

Les observations formulées jusqu'ici portaient sur les propriétés linguistiques des items décrits. Les composants étymologiques doivent en outre permettre de situer dans le temps les entrées en usage des mots.

De véritables enseignements d'histoire figurent aux programmes scolaires à partir du cycle 3 . Âgés de 8 ans et plus, les enfants doivent acquérir une représentation des durées inscrites dans le passé en abordant une vingtaine de «points forts 》 (Qu'apprend-on à l'école élémentaire, $2002: 210$ ) répartis de la préhistoire au $\mathrm{XX}^{\mathrm{e}}$ siècle (pp. 209-215) et en situant « quelques personnages majeurs dans l'ordre politique, mais aussi littéraire, artistique ou scientifique » (p. 209). La chronologie relative des périodes passées et des événements historiques qui les jalonnent est en cours d'acquisition et c'est sur elle que les descriptions étymologiques vont pouvoir s'appuyer pour expliquer que la langue française n'a pas toujours été ce que l'on en connaît et qu'elle s'est enrichie au rythme de l'histoire de ses locuteurs. Il y aurait donc une certaine logique à permettre aux jeunes consultants de construire des repères qui pourraient faire écho à des événements découverts en cours d'histoire, ou dans d'autres matières d'éveil (géographie, biologie, physique, etc.), ou hors des établissements scolaires. Ce n'est pourtant pas ce qui est privilégié dans la majorité des descriptions étymologiques étudiées. Comme le montrent les données présentées ci-après, les repères chronologiques fournis (mentions d'années ou de siècles, référence à des événements réputés connus ou présentés en contexte afin de les situer), quand ils existent, ne sont pas toujours utilisables en confiance pour dater les usages linguistiques.

\subsection{Premières attestations et entrée en usage des mots}

La majorité des étymologies des dictionnaires scolaires ne fournissent pas de repères chronologiques. Peut-être est-ce pour contourner les difficultés que les enfants pourraient encore rencontrer à situer une date ou une période historique. Quelques autres réfèrent à des réalités anciennes situées dans le temps de manière très vague. C'est le cas par exemple s.v. calcul (cf. [14]), pour lequel le $L S M$ justifie le cheminement depuis calculus, l'étymon latin cité, par le fait que « autrefois, on utilisait des cailloux pour compter. », sans que autrefois puisse permettre de comprendre que cet usage remonte à deux millénaires. Rien ne semble justifier cette localisation floue préférentiellement à une référence à l'Antiquité romaine, à défaut de repérage plus précis.

Le RJI-CE, le seul dont les articles sont dédiés à l'étymologie, fournit seulement quinze datations de mots (quatorze noms et un adjectif). Les deux tiers d'entre eux ont intégré le vocabulaire français depuis la fin du XVIII ${ }^{\mathrm{e}}$ siècle, un l'avait fait au XVII' et les autres au Moyen Âge. ${ }^{29}$ Cet ensemble ne permet pas aux consultants d'imaginer l'importance relative des siècles qui ont précédé le $\mathrm{XVIII}^{\mathrm{e}}$, alors que la sélection des mots dont l'histoire est décrite dans ce cahier permettrait tout à fait de montrer explicitement les fonds les plus anciens et les vagues d'enrichissements les plus significatives : après recherche des dates des premières attestations de chaque item dans des sources externes ( $D H L F$ et $T L F i$ en particulier), il semble que seuls les apports germaniques enregistrés entre le $\mathrm{V}^{\mathrm{e}}$ et le $\mathrm{X}^{\mathrm{e}}$ siècle (blé, guerre, par exemple) ne soient 
pas représentés. La couverture chronologique des « 400 histoires de mots » (RJI-CE: $1^{\mathrm{e}}$ de couverture) bien qu'imperceptible par les consultants, puisque seulement $4 \%$ d'entre elles localisent dans le temps les attestations lexicales, est donc importante.

Pour le même public, le $D M D$ et le $L S M$ racontent les grandes étapes de l'évolution de la langue française dans des hors-texte.

- Le $D M D$ le structure en cinq parties : «Les Gaulois et la conquête romaine », «Vers l'ancien français », "Le français au Moyen Âge », «L'enrichissement du français grâce au latin et au grec », "Les mots étrangers devenus français ». Dans les limites imposées par sa rédaction sur cinq pages (cf. supra, introduction), il articule l'histoire de la langue et celle du peuple en se concentrant principalement sur les périodes les plus anciennes : celles qui sont abordées en premier en cours d'histoire ${ }^{30}$, qui correspondent à une société très différente de ce qu'elle est aujourd'hui et qui ont vu le français se constituer par héritages puis emprunts de mots aux formes souvent malaisées à identifier et par constructions de mots nouveaux à partir de constituants empruntés ou français. Bien qu'il fournisse des exemples de mots intégrés à plusieurs des époques décrites, ce paratexte ne trouve pas d'écho dans les articles du dictionnaire, qui ne proposent aucune étymologie (cf. n. 5). Ce texte diachronique ancre les étapes de l'élaboration de la langue française par l'évocation (i) de deux événements, (ii) de trois périodes nommées et (iii) de trois périodes localisées par des siècles :

(i) «Après la conquête de la Gaule par le général romain César (au milieu du $1^{\text {er }}$ siècle avant notre ère), les Gaulois adoptent peu à peu la langue des Romains, c'est-àdire le latin. $»^{31}$ (p. 1064) et « $\mathrm{Au}$ cours des mille ans qui suivent la conquête romaine, le latin populaire parlé en Gaule change beaucoup. » (p. 1065) ; "C'est surtout à partir de la Révolution que la proportion de mots d'origine anglaise s'accroît » (p. 1068);

(ii) « Au cours du Moyen Âge et de la période qui a suivi (en particulier au $16^{\mathrm{e}}$ siècle, pendant la Renaissance), des traducteurs et des écrivains ont créé en grand nombre de nouveaux mots français en prenant, sans presque les changer, des mots latins et des mots grecs empruntés aux écrivains de l'Antiquité. » (p. 1066) ; «Dès le Moyen Âge, le néerlandais a donné au français une grande partie des mots liés au thème de la mer» $\gg($ p. 1067);

(iii) « La langue des peuples germaniques qui ont envahi la Gaule dans les premiers siècles de notre ère, a donné de nombreux mots » (p. 1067); «L'espagnol et le portugais, à la suite de la découverte de l'Amérique au $16^{\mathrm{e}}$ siècle, ont fourni beaucoup de noms d'animaux ou de denrées venues de ces contrées» (p. 1068); «l'anglais qui, depuis le $11^{\mathrm{e}}$ siècle, fournit un important stock de vocabulaire au français. » (p. 1068).

Il situe le(s) premier(s) siècle(s) avant notre ère (pp. 1064 et 1067), mentionne deux siècles (XI ${ }^{\mathrm{e}}, \mathrm{p} .1067$, et $\mathrm{XVI}^{\mathrm{e}}$, pp. 1066 et 1068, écrits en chiffres arabes) et ne cite aucune date précise. Cependant, trois dates jugées importantes sont présentées dans ses marges accompagnées de très courts extraits reproduits et traduits de textes de ces époques: «842», Serments de Strasbourg; «881», Cantilène de Sainte Eulalie; et « vers 1200 », un poème en langue d'oc de Bertran de Born et un en langue d'oül de Chrétien de Troyes. Si les articles de ce dictionnaire recevaient des étymologies, des mentions d'œuvres de ce type permettraient aux consultants de percevoir à la fois la rareté des textes anciens (peu de titres peu mentionnés) et la justification des datations de mots que pourraient offrir leurs descriptions étymologiques.

- Le LSM fournit, lui, un texte de présentation historique très dense (cf. supra, introduction) et des étymologies dans une sélection importante d'articles du dictionnaire. Le hors-texte ne situe pas dans le temps les conquêtes romaines mentionnées dans son introduction, mais il localise (p. 8) d'autres événements anciens : les invasions germaniques ( $\mathrm{A}$ partir du III siècle après J.-C. »), la fin de l'Empire romain d'Occident (« en $476 »)$ et les « défaites des Wisigoths d'Alaric en 507 [et] des Burgondes en 534 ». La séparation des parlers d'oc et d'oül est également située (p. 9) « [a]u cours du Moyen Âge, à partir du IX ${ }^{\mathrm{e}}$ siècle » ainsi que quelques textes jugés importants : «Les serments de Strasbourg (842)»32 et « l'ordonnance de Villers-Cotterêts signée par François $1^{\mathrm{er}}$ en $1539 » .{ }^{33}$ Ce sont ensuite certaines périodes d'échanges qui sont localisées dans le temps (p. 11), comme ceux entretenus avec les langues italienne et proven- 
çale (« dès le XIII ${ }^{\mathrm{e}}$ siècle » puis avec « la renaissance des foires marchandes et de change au XV" siècle » et avec « une véritable mode «italianizzante» [qui] s'est répandue en France au XVI siècle »), avec les peuples arabes et espagnols (les premiers étant « en Espagne du VIII ${ }^{\mathrm{e}}$ au XV $\mathrm{XV}^{\mathrm{e}}$ siècle » et « dans le sud-ouest de la France jusqu'en 759 ») et avec la langue anglaise (à partir du " $\mathrm{XI}^{\mathrm{e}}$ siècle, le français a été, jusqu'au milieu du XIV ${ }^{e}$ siècle, la langue de la cour royale d'Angleterre », «[p]uis les mots sont revenus à leurs expéditeurs. [...] budget, emprunté à l'anglais au XVIII ${ }^{\mathrm{e}}$ siècle [...] cabinet $[. .$.$] revenu au XVII { }^{\mathrm{e}}$ siècle $\left.»\right)$. Pour la sélection d'événements ou de périodes situés dans le temps, des datations précises ont été fournies quand cela était possible, ce qui fait qu'elles côtoient des repérages par siècles (notés en chiffres romains), chacun des deux types d'expression semblant choisi selon son adéquation locale plus qu'en fonction des connaissances supposées des consultants. Ces repères sont cependant trop peu nombreux et systématiques et il est regrettable que les informations présentées dans la «chronologie universelle» (pp. 1295-1326) et qui ont une incidence linguistique n'aient été que minoritairement reprises dans la « Petite histoire de la langue française » et, pour celles qui le sont, que leurs mentions dans les deux hors-texte ne soient pas liées. Enfin, il est dommage que les étymologies des articles ne renvoient pas aux hors-texte et que les lecteurs ne puissent pas ancrer les données qu'elles fournissent dans les repères qu'ils proposent.

\subsection{Localisation des emplois des mots par rapport à des événements}

Durant différents cours ${ }^{34}$ et dans diverses ressources non pédagogiques, d'autres repères chronologiques peuvent être fournis. Ils concernent par exemple les premières importations de certains produits qui ont intégré notre culture consécutivement à la découverte et/ou à la conquête de nouveaux territoires ou à l'instauration de nouvelles voies de commerce. Ces objets, nommés dans la langue de leur pays d'origine ou dans celle de leurs exportateurs, ne l'étaient pas nécessairement encore en français mais ils l'ont été très vite s'ils ont connu une certaine diffusion. Par conséquent, la datation de l'introduction en France des produits peut fournir un repère certes approximatif mais culturellement ancré. Dans le RJI-CE, s.v. chocolat, banane et yaourt, les découvertes et diffusions des référents sont racontées pour motiver l'emploi de ces mots en français, mais la qualité des repères chronologiques fournis n'est pas aussi bonne partout.

S.v. chocolat (cf. [15]), il est indiqué que ce sont les mariages des rois Louis XIII et Louis XIV avec des infantes d'Espagne qui ont permis à la cour de France de connaître la boisson et le mot, ce qui situe dans la première moitié du XVII ${ }^{\mathrm{e}}$ siècle un repère temporel que corroborerait en l'affinant la consultation d'encyclopédies (mariage de Louis XIII en 1615 avec Anne d'Autriche, qui contribua la première à la diffusion du chocolat en France, monopole accordé à une manufacture de chocolat par Louis XIV en 1659, un an avant son mariage) et que recoupent assez bien les datations du DHLF (1598 pour chocolate, 1659 pour chocolat).

[15] s.v. chocolat ${ }^{35} \cdot$ «Ce mot vient de l'espagnol chocolate; c'est l'adaptation d'un mot de la langue d'un ancien peuple indien du Mexique qui signifiait peut-être « eau amère ». Les Aztèques accordaient une grande valeur à ce breuvage des dieux et le cacaoyer était un arbre sacré. Les conquérants espagnols prennent goût au chocolat et expédient des fèves de cacao vers l'Espagne. Louis XIII et Louis XIV épousent des infantes d'Espagne qui font connaitre la nouvelle boisson à la cour de France qui adopte le produit et le mot. » $(R J I-C E)$

Concernant banane (cf. [16]), la seule datation indiquée est le XVI siècle, ce qui correspond au moment où les Portugais, qui avaient découvert les bananiers en Afrique, les répandirent aux Caraïbes et en Amérique. S'il est écrit que le mot français est emprunté à la langue portugaise, rien n'indique que la diffusion du fruit en Europe date du XVI ${ }^{\mathrm{e}}$ siècle. La dernière phrase de la description incite plutôt à penser que le fruit était arrivé en France antérieurement, mais, comme il a d'abord porté un autre nom, l'incertitude demeure sur l'époque de l'apparition du mot banane. Le DHLF signalant des attestations pour le mot portugais banana en 1562 et pour les formes françaises bannanas en 1598 et banane en 1602, l'évocation du $\mathrm{XVI}^{\mathrm{e}}$ siècle par le $R J I-C E$ constitue bien un repère valide pour dater le mot, mais son caractère indirect fait que les consultants du dictionnaire ne peuvent avoir aucune certitude à ce sujet.

[16] s.v. banane ${ }^{36} \cdot$ «La banane est un fruit originaire d'Asie. Le bananier est acclimaté en Afrique et sa culture progresse de l'est du continent vers l'ouest. Les Portu- 


\begin{abstract}
gais y découvrent cette plante inconnue des Européens. Au $16^{\mathrm{e}}$ siècle, ils la répandent aux Caraïbes et en Amérique. Ce mot vient du portugais banana, qui vient probablement d'un mot africain de Guinée. Le fruit était connu auparavant mais on le nommait en français pomme de paradis. » (RJI-CE)
\end{abstract}

$\mathrm{Si}$, dans les deux cas précédents, les repères chronologiques fournis concernant les référents coïncidaient approximativement, quoique de façon inégalement explicite, avec l'introduction des noms en français, la date fournie s.v. yaourt (cf. [17]) ne doit pas pousser les consultants à croire que le mot a été introduit à cette époque. La mention des noms commerciaux doit les préserver de cette erreur, mais le fait que la phrase qui les cite soit suivie d'une autre plus strictement étymologique pourrait les inciter à croire que les formes yogourt et yaourt n'ont été attestées en français qu'après la commercialisation du produit sous des noms orientaux, alors qu'elles l'ont été respectivement en 1432 et 1798-1799 selon le DHLF.

[17] s.v. yaourt ${ }^{37} \cdot$ «Cette préparation à base de lait fermenté est consommée traditionnellement dans les Balkans, en Asie centrale et surtout en Turquie et en Bulgarie. Dans les années 1920, ce produit est commercialisé en France, principalement dans les pharmacies, sous des noms qui évoquent son origine orientale (Pacha, Constantinople, Sofia, Caucase...). Ce mot vient du turc yogurt; en français, on trouve d'abord yogourt, et, plus tard, yaourt. » (RJI-CE)

\title{
4.3 Situation et motivation des dérivations de mots à partir de noms propres
}

Enfin, une part non négligeable des descriptions étymologiques portent sur des mots créés à partir de noms propres de lieux ou de personnes. Pour ces mots, il faut non seulement expliquer quel a été leur mode de production et dans quelle langue (pasteuriser, cf. [18], est construit en français, il résulte de la suffixation en -is(er) de Pasteur; sandwich, cf. [19], vient de l'antonomase en anglais du nom du comte Sandwich ; bégonia, cf. [20], est le produit de la suffixation en latin botanique de -ia au nom d'un intendant de marine français nommé Bégon), mais il importe également d'en motiver la création (dénommer respectivement un procès en référence à des préconisations d'une personne notoire (pasteuriser), une entité en référence à une personne à laquelle elle est associée et qui tire sa notoriété de cette dénomination (sandwich), et une espèce végétale en hommage à une personne à qui, le plus souvent, la botanique doit quelque chose $\left.(\text { bégonia })^{38}\right)$, ce qui implique des évocations historiques ayant leur ancrage chronologique propre, qui peut être ou ne pas être en synchronie avec l'entrée des mots correspondants dans le lexique français. L'observation des traitements de ces mots proposés par les quatre dictionnaires scolaires examinés conduit à constater qu'ils sont plus à l'aise dans les évocations historiques (où le $R J I-C E$ se met particulièrement en vedette) que dans la synchronisation de celles-ci avec la datation des faits lexicaux, ce qui rejoint les observations faites ci-dessus à propos de chocolat, banane et surtout yaourt.

S.v. pasteuriser, aucun des dictionnaires ne signale la suffixation en -is(er) du nom de Louis Pasteur, alors que chacun motive la création de ce verbe : le $L S M$ invite très implicitement ses lecteurs à consulter l'article Pasteur ${ }^{39}$ dans sa section réservée aux noms propres ; les Hachette fournissent un minimum d'informations ; le RJI-D date la mise au point de la pasteurisation sans expliquer en quoi elle consiste; le $R J I-C E$, plus didactique, présente l'homme et le procédé qu'il a préconisé dans l'article du verbe qui exprime la réalisation du procès. Tous les répertoires évoquent, s.v. pasteuriser, l'homme et, localement ou s.v. Pasteur, sa découverte, aucun ne date la création du verbe (1872) ou celle du nom (1887).

[18] s.v. pasteuriser • «Pasteuriser vient du nom de Louis Pasteur, biologiste français du XIX ${ }^{\mathrm{e}}$ siècle, qui inventa ce procédé de conservation. » $(D H J) \bullet$ «Pasteuriser vient du nom de Louis Pasteur, chimiste et biologiste français du XIX ${ }^{\mathrm{e}}$ siècle, qui inventa ce procédé de conservation. » $(D S H)$ - «Vient du nom de Louis Pasteur. » $(L S M) \bullet ~ « C e$ mot vient du nom du savant Louis Pasteur, qui a mis au point cette méthode vers $1860 . »(R J I-D) \bullet$ «Ce mot vient d'un nom propre. Louis Pasteur est un chimiste du $19^{\mathrm{e}}$ siècle qui découvre que la fermentation est provoquée par des organismes microscopiques. Si l'on détruit les germes et les bactéries contenus dans un liquide comme le lait, le cidre ou la bière, on peut le conserver plus longtemps et il n'est pas dangereux à consommer. Pasteur a recommandé de chauffer et de refroidir 
Concernant des dérivés de noms d'hommes moins illustres (cf. [19]), comme par exemple le Comte Sandwich, les dictionnaires présentent le personnage et souvent son occupation favorite, le $L S M$ y ajoute une datation de la création du premier en-cas du type de ceux qui porteront ce nom et le $R J I-C E$ indique que ce comte vécut au XVIII ${ }^{\mathrm{e}}$ siècle, qu'il était anglais (ce qui explique que le $R J I-D$ et les Hachette signalent que ce mot est un anglicisme ${ }^{40}$ ) et que son nom a également été donné à un $\operatorname{archipe}^{41}$ (ce qui n'a aucune importance concernant l'emploi du nom commun en français). Aucun dictionnaire ne date l'emploi du nom pour désigner l'en-cas ni en anglais ni en français (respectivement 1762 et 1802 selon le DHLF).

[19] s.v. sandwich • « Sandwich vient du nom du comte de Sandwich ${ }^{42}$, dont le cuisinier inventa ce repas sommaire pour lui éviter de quitter sa table de jeu. » (DHJ et $D S H) \bullet$ «Vient du nom du comte de Sandwich, pour qui un cuisinier inventa cet encas en 1762, afin de lui permettre de manger sans quitter la table de jeu.» $(L S M)$ - «C'est un mot anglais, qui vient du nom du comte de Sandwich.» $(R J I-D) \bullet$ «Ce mot vient d'un nom propre. Un Anglais du $18^{\mathrm{e}}$ siècle, John Montagu, $4^{\mathrm{e}}$ comte de Sandwich, était passionné par le jeu. Il ne souhaitait même pas interrompre la partie en cours pour prendre un repas! Son cuisinier glissa de la viande entre deux tranches de pain et ce repas sommaire devint à la mode sous le nom de sandwich. Le nom de ce même personnage est attaché à un archipel. [...]. » (RJI-CE)

Comme le nom propre de l'archipel hawaïen, certains noms communs sont des dédicaces. Ainsi en va-t-il pour bégonia, introduit en France et nommé par le botaniste Plumier en l'honneur de l'intendant Bégon. Le RJI-CE fait ce récit mais il ne date pas la première attestation du nom (avant 1706 pour la forme begonia selon le $D H L F$ ), les Hachette localisent l'entrée de la plante en France («à l'époque de Louis XIV ») mais pas l'emploi du nom et ils négligent la dédicace.

[20] s.v. bégonia • «Bégonia vient du nom de Michel Bégon, qui ramena cette plante de Saint-Domingue à l'époque de Louis XIV.» $(D H J$ et $D S H) \bullet$ "Ce mot vient du nom de Michel Bégon, créateur d'un jardin botanique. » $(R J I-D) \bullet$ « Ce mot vient d'un nom propre. Michel Bégon, intendant de marine, crée en 1697 à Rochefort (en Charente-Maritime) un jardin botanique destiné à la culture des plantes exotiques et il envoie une expédition étudier la flore des Antilles. Un botaniste en rapporte une plante fleurie inconnue en France ; il l'appelle bégonia, en l'honneur de l'organisateur de l'expédition. » $(R J I-C E)$

\subsection{Orientations conclusives pour l'ancrage de l'histoire de mots dans une chronologie accessible}

L'évocation d'événements découverts ailleurs doit permettre aux jeunes lecteurs d'établir des liens et, compte tenu de leur âge, il est utile de les inviter à consulter d'autres ressources, dont en particulier des articles d'encyclopédies ou de dictionnaires de noms propres. Les renvois peuvent alors être explicites, entre parties d'un même volume ou ouvrages d'un même éditeur, s'il permettent de localiser dans le temps les éléments fournis - les vies de Louis XIII et Louis XIV pour [15], celle de Pasteur pour [18] ou si les articles cibles sont rédigés de manière articulée, ce qui ne sera pas toujours aussi aisé - la pasteurisation est importante pour Pasteur mais l'introduction du chocolat n'est certainement pas un fait majeur des règnes des rois Louis XIII et Louis XIV. En tout état de cause, la modestie supposée de la culture historique des lecteurs doit inciter à la précision locale et pousser à fournir la datation des emplois linguistiques et celle des événements qui sont évoqués de manière à permettre à chacun de tirer le meilleur profit de sa lecture. Elle pourrait également encourager à référer chaque événement à une synthèse diachronique paratextuelle. ${ }^{43}$ Cette synthèse pourrait, comme la « chronologie universelle » du LSM, localiser une sélection d'événements historiques et artistiques, mais il serait souhaitable que son échelle de rédaction et la modularité de son affichage permettent d'y repérer ceux qui sont évoqués dans chacun des articles et un choix d'autres jugés marquants. Dans une version électronique, ceci consisterait à afficher le monopole accordé à une manufacture de chocolat (1659) et le mariage de Louis XIV (1660) lorsque la chronologie est consultée à partir de l'article chocolat, s'il évoque bien ces événements, et à ne retenir autrement que 
la signature du traité des Pyrénées (1659) et la mort du Cardinal Mazarin (1661), qui marquent plus notoirement ces années. Les articles agitateur et agiter et les occurrences de ces items dans le schéma de la famille d'agir (cf. annexe 2) pourraient, dans cette chronologie, permettre d'accéder aux événements majeurs de la Révolution française, qui jalonneraient stablement cette période, et aux principaux actes de Robespierre afin de situer le discours qu'il a formulé et qui contient la première attestation d'agitateur ${ }^{4}$.

Les orientations rédactionnelles présentées ci-dessus et en annexes accordent un intérêt particulier aux datations, ce qui impose de revenir sur l'évocation (au § 4.1) de la fragilité des compétences des consultants qui va influer sur l'écriture. L'alternative est la suivante : (i) l'accumulation de datations précises (comme 1874 s.v. impressionniste dans le $R J I-C E$ ), outre que le plus souvent cette précision aurait un caractère illusoire (les datations ne repérant que des attestations et non la diffusion des usages), pourrait nuire à la prise de repères chronologiques plutôt que de la soutenir ; (ii) une localisation par siècles (ou demi-siècles) éviterait cet écueil, mais ces repères pourraient ne pas être intelligibles par les consultants les plus jeunes, la notion de siècle n'étant introduite qu'au cycle 3, avec le début des enseignements d'histoire. Localiser aussi souvent que possible, comme cela est fait pour agitateur ci-dessous, les premières attestations en mentionnant simultanément les siècles en chiffres romains et les dates écrites en chiffres arabes devrait permettre de nuancer les datations et de consolider le déchiffrage et le repérage des siècles.

Le français a emprunté le nom anglais agitator (“celui qui agite les esprits et cause le trouble") à la fin du XVIII ${ }^{\mathrm{e}}$ siècle en modifiant légèrement sa forme finale (-or en anglais / -eur en français) mais pas son sens. Ce nom masculin a été employé pour la première fois en 1792 par Robespierre, dans un discours prononcé devant les Jacobins.

Ce principe conduit en outre à valoriser les textes sources, ce qui peut être apprécié dans le cas présent ou pour acte ("subdivision d'une pièce de théâtre") attesté dans un essai de Montaigne", qui sera certainement souhaité pour les items figurant dans les premiers textes écrits en langue française (comme plaisir (cf. § 1.2) attesté dans la Chanson de Roland en 1080), mais qui peut sembler moins voire non pertinent quand il s'agit de textes méconnus comme ceux où figure agenda (cf. nn. 48 et 49).

\section{Conclusion}

Les dictionnaires actuels décrivent environ 20000 mots français pour le cycle 3 et quelques milliers de plus pour le collège. Au sein de ces nomenclatures, les items présentent une telle diversité de procédés de création, d'origines et de modes d'intégration au lexique français qu'ils fournissent une matière trop abondante pour être exploitée pleinement dans le cadre d'une première sensibilisation à l'étymologie. En sélectionnant les mots auxquels sont associées des informations étymologiques, les lexicographes peuvent privilégier différents aspects : ludique, en cherchant à surprendre les consultants ; anthropologique, en choisissant des mots (souvent de la vie courante) empruntés à un large ensemble de langues et donc de populations ; concret, en accordant une préférence à des mots dont l'origine est indissolublement liée à celle d'objets dont la circulation est internationale ; historique, en signalant des mots que le français a intégrés consécutivement à des événements notoires; culturel, en retenant des mots associés à des découvertes jugées primordiales; etc.

Quelles que soient les motivations présidant au choix des articles dotés d'une information étymologique, celle-ci doit avant tout fournir des données relatives à l'histoire du mot et de ses usages. Certaines sont naturellement le prétexte à des enrichissements non linguistiques : les mentions de langues d'origine qui incitent à évoquer les locuteurs de ces langues et la manière dont leur parler est entré en contact avec des francophones, les datations d'entrées de mots dans notre lexique qui amènent à faire mention de faits historiques ou scientifiques. Ces enrichissements sont nécessaires pour fonder la notion d'histoire de la langue, mais leur évocation fragmentée dans les composants étymologiques ne suffit pas pour établir une chronologie linguistique intelligible. Le $D M D$ et le $L S M$ sont les seuls dictionnaires scolaires à proposer des paratextes historiques, mais leur échelle de rédaction les rend moins didactiques que ceux d'ouvrages proprement étymologiques destinés à des lecteurs d'âge scolaire, comme l'introduction du petit répertoire Les mots vagabonds (De Hugo 2003), qui présente les circonstances dans lesquelles douze langues ou groupes de langues ont contribué à enrichir le français (pp. 7-13), ou le texte de l'album Le français. Les 
mots voyageurs (Thévenin 1986), qui offre un panorama historique d'une quarantaine de pages richement doté de mots illustrant chaque vague d'enrichissement linguistique présentée. La rédaction d'une synthèse historique de qualité pourrait s'appuyer sur différents textes qui vulgarisent des données linguistiques, comme les encadrés du DHLF, l'introduction du Dictionnaire des mots d'origine étrangère (Walter \& Walter 1991 : 17-115), la description de l' «origine des mots » dans Le bon usage (Grevisse \& Goosse 2008 : 150-213) ou encore des manuels universitaires (Picoche \& Marchelo-Nizia (1998) et Perret (2008) par exemple) et sur les programmes des matières d'éveil ou leur mise en œuvre dans les ouvrages scolaires (livres et cahiers d'exercices des élèves et livres du maître (cf. nn. 30 et 34)), qui fourniraient les faits et notions qui seront vraisemblablement abordés et qui pourraient donc être utilisés pour baliser la chronologie historique. Cette rédaction devrait en outre être articulée à celle des articles du répertoire de manière à ce qu'elle puisse tenir compte de la nomenclature du dictionnaire qu'elle intégrerait (pour ne présenter, comme illustration de son propos, que des mots décrits, voire, et ce serait mieux, que des mots pour lesquels des informations étymologiques seront fournies) et des repères extralinguistiques exploités dans les articles, ce qui permettrait de poser que tout ce qui est dans les composants étymologiques peut être resitué dans la chronologie synthétique du paratexte. Elle pourrait enfin être secondée par des horstexte qui relateraient quelques histoires de familles de mots (cf. annexe 2), ce qui permettrait de remédier à la pratique actuelle consistant à les présenter dans le composant étymologique d'un mot particulier alors qu'elles mériteraient d'être décrites au titre de chacun (cf. § 2). Ce dispositif donnerait par ailleurs la possibilité de spécifier que certains des mots d'une famille sont employés depuis plusieurs siècles alors que d'autres, qui actuellement peuvent être signalés dans un composant morphologique synchronique des articles, y ont été plus récemment intégrés.

Enfin, en leur qualité d'outils de consolidation de la compétence linguistique, il est capital que les dictionnaires aident à repérer les propriétés phonographiques, morphologiques et sémantiques des mots en usage actuellement. Là encore les informations étymologiques peuvent être exploitées pour éclairer le présent en expliquant le passé. En conséquence, même si les enfants du cycle 3 et du collège ne doivent pas apprendre toutes les règles qui ont permis aux mots du fonds latin d'évoluer pour constituer le français moderne, il est important de leur indiquer que les formes des mots ont changé et de les sensibiliser à ces évolutions en leur en présentant les mécanismes les plus récurrents dans un paratexte didactique auquel renverraient les composants étymologiques des articles. La perception des évolutions phonographiques et des principes de construction des mots qui, en français ou dans d'autres langues, ont permis de produire ceux qui sont décrits dans les composants étymologiques permettrait aux jeunes consultants d'avoir une intuition sur l'histoire d'autres mots pour lesquels aucune information étymologique ne leur est fournie, de décoder spontanément le sens de néologismes ou de mots anciens mais découverts par eux, par exemple, en cours d'éveil, de mémoriser les particularités phonographiques de certains mots en sachant les motiver et de créer, avec plus de confiance puisqu'en connaissant certains patrons valides, les mots non encore existants ou connus d'eux dont ils ont besoin pour s'exprimer. Il est donc capital, autant que cela est possible, de préciser dans quelle langue chaque étape de construction ou d'évolution est survenue, de nommer les mécanismes en œuvre de manière à les rendre identifiables et, pour les dérivations ou compositions, de repérer chaque constituant impliqué en mentionnant, si c'est pertinent, plusieurs de ses formes et en le glosant. Ces indications fournies pour une sélection de mots, ceux dont les articles intègrent une étymologie, pourraient par ailleurs utilement être articulées à un paratexte (cf. n. 21) qui présenterait les règles de manière synthétique, un peu à la manière de ce que propose le $D M D$ (cf. supra, introduction) mais en l'enrichissant d'observations qui contrasteraient les mécanismes du français moderne avec ceux d'états de langue plus anciens et d'autres langues (anciennes ou modernes) afin de sensibiliser aux proximités et différences du latin, des langues romanes et de l'anglais au moins.

Les pistes de réflexion sont multiples, mais elles visent toutes à systématiser la présence des composants informationnels et à les rendre les plus explicites possible, de manière à ce que localement le texte de chaque étymologie puisse être pleinement compris et qu'avec le soutien de paratextes historiques et morphologiques les consultants puissent situer dans un cadre global les informations fournies pour chaque mot. Pour mieux évaluer la faisabilité d'un dictionnaire historique pour les jeunes qui prendrait en compte ces orientations, il conviendrait maintenant ${ }^{46}$ d'en établir la charte rédactionnelle et de l'appliquer à un échantillon représentatif de mots, ce qui constituerait à soi seul la matière d'un autre texte. 


\section{Références bibliographiques}

De Hugo, C. (2003). Les mots vagabonds. Ces mots français venus d'ailleurs. Paris : Mango Jeunesse.

DFV = Davau, M., Cohen, M. \& Lallemand, M., (1971). Dictionnaire du français vivant. Paris : Bordas. - Nouv. éd. entièrement revue et augmentée. 1980.

DHJ = Dictionnaire Hachette junior. (2006). Paris : Hachette Éducation.

$D H L F=$ Rey, A., dir. (1998). Dictionnaire historique de la langue française. Éd. enrichie. 3 vol. Paris : Dictionnaires Le Robert.

$D M D=$ Dictionnaire Maxi débutants. (1999). Paris : Larousse.

DSH = Dictionnaire scolaire Hachette. (2002). Paris : Hachette Éducation. ${ }^{47}$

Gasiglia, N. (2008). Le traitement des emprunts dans les dictionnaires d'apprentissage français : options descriptives et choix rédactionnels. In J. Pruvost (dir.), Les Journées des dictionnaires de Cergy. Dictionnaires et mots voyageurs. Les 40 ans du Petit Robert. Collection Actes de colloque. Eragny-sur-Oise : Éditions des Silves. 153-212.

Grevisse, M. \& Goosse, A. (2008). Le bon usage. Grammaire française. $14^{\mathrm{e}}$ éd. revue. Bruxelles : De Boeck Duculot.

Hausmann, F.J. \& Wiegand, H.E. (1989). Component parts and structures of general monolingual dictionaries: a survey. In F.J. Hausmann, O. Reichmann, H.E. Wiegand \& L. Zgusta Hrsg., Wörterbücher / Dictionaries / Dictionnaires. Ein internationales Handbuch zur Lexikographie / An International Encyclopedia of Lexicography / Encyclopédie internationale de lexicographie, t. 1, art. 36. Berlin / New York : Walter de Gruyter. 328-360.

Le Callennec, S., dir. (2007a). Histoire Géographie CE2 Cycle 3 conforme aux nouveaux programmes. Guide pédagogique. Collection Magellan. Paris : Hatier.

Le Callennec, S., dir. (2007b). Histoire Géographie CM1 Cycle 3 conforme aux nouveaux programmes. Guide pédagogique. Collection Magellan. Paris : Hatier.

Le Callennec, S., dir. (2007c). Histoire Géographie CM2 Cycle 3 conforme aux programmes 2002. Guide pédagogique. Collection Magellan. Paris : Hatier.

Le Grand dictionnaire Hachette-Oxford. (2003). $3^{\mathrm{e}}$ éd. CD-ROM PC. Oxford : Oxford University Press / Paris : Hachette Mutimédia.

Le Grand Robert \& Collins électronique. (2003). CD-ROM PC, version 1.0. Paris : Dictionnaires Le Robert - VUEF / Bruxelles : Bureau van Dijk.

LSM = Larousse Super Major. (2004). Paris : Larousse.

Perret, M. (2008). Introduction à l'histoire de la langue française. $3^{\mathrm{e}}$ éd. Collection Cursus. Paris : Armand Colin.

Picoche, J. \& Marchello-Nizia, C. (1998). Histoire de la langue française. $5^{\mathrm{e}}$ éd. Collection fac. Paris : Nathan.

Qu'apprend-on à l'école élémentaire? Les nouveaux programmes. (2002). Paris : Centre National de Documentation Pédagogique / XO Éditions.

$R J I=$ Le Robert Junior illustré. (2003). Paris : Dictionnaires Le Robert. - Nouv. éd. entièrement revue et enrichie. (2005).

RJNP $=$ Le Robert Junior des noms propres. (2005). Paris : Dictionnaires Le Robert.

Thévenin, A. (1986). Le français. Les mots voyageurs. Paris : Épigones.

$T L F i=$ Trésor de la langue française informatisé. (2004). Cédérom du texte intégral, versions PC et Mac OS. Paris : CNRS Éditions.

Walter, H. \& Walter, G. (1991). Dictionnaire des mots d'origine étrangère. Paris : Larousse.

\section{Annexe 1 : Patron de rédaction de l'article agenda}

Le patron de rédaction qui est ébauché pour l'article agenda ci-dessous est respectueux des principes rédactionnels introduits précédemment dans les « orientations conclusives » (cf. §§ 2.8, 3.4 et 4.4). Il permet d'exposer un premier état de la sélection des informations à présenter, mais sa rédaction n'est pas aboutie et elle ne peut pas l'être sans 
définir des paramètres éditoriaux comme l'interface de consultation (vraisemblablement électronique pour la malléabilité d'organisation textuelle qui la caractérise et pour sa plausible attractivité auprès du public visé). Ces choix devront assurer le décodage des segments soulignés (i) en définissant les termes qui n'appartiennent pas à la métalangue scolaire la mieux partagée et les noms de langues ou d'états de langue avec, pour les derniers, des renvois vers une chronologie situant leur période d'emploi ; (ii) en présentant les textes associés aux datations. Ces choix devront également permettre d'adopter un mode rédactionnel au moins aussi explicitant que celui proposé ci-dessous et de présenter le texte de manière modulaire (texte complet intégral (comme ci-dessous) / minimum intégral (non fourni) / minimum avec des modules ( $\mathrm{a}, \mathrm{b}, \mathrm{c}$ ou d) complets).

\section{[1'] s.v. agenda}

(a) En bas latin existait le verbe agere ("faire"). Une de ses formes exprimait l'obligation future de réaliser le procès qu'il exprimait. Cette forme verbale a été convertie en un adjectif, agendus, qui exprimait aussi l'obligation future : "qui doit être fait". L'adjectif agendus a lui-même été converti en nom : agenda "choses à faire".

- Des conversions en chaîne sont également observables en français. Par exemple le verbe gagner a permis de construire l'adjectif gagnant qui a permis de construire le nom : Avais-tu parié sur le cheval qui a gagné ${ }_{[\mathrm{V}]} l a$ course?; Avais-tu parié sur le cheval gagnant ${ }_{[\mathrm{A}]}$ ?; Avais-tu parié sur le gagnant ${ }_{[\mathrm{N}]}$ de la course?

(b) En latin ecclésiastique, en France à la fin du XIII ${ }^{\mathrm{e}}$ siècle, on employait l'expression agenda diei qui signifiait "registre des offices (services religieux) du jour".

Le français a emprunté le nom latin agenda au XVI ${ }^{\mathrm{e}}$ siècle sans en modifier la forme mais en en faisant un nom masculin singulier. On le trouve écrit en 1535 dans un texte peu connu ${ }^{48}$. À l'époque de ce texte, ce nom a servi à désigner le "livre des comptes d'une ville". À partir du XVII ${ }^{\mathrm{e}}$ siècle, il a permis de nommer le "registre dans lequel sont consignées les choses à faire". Ce sens est attesté en 1662, cette fois encore dans un texte peu connu $^{49}$.

(c) $\rightarrow$ Le verbe agir et d'autres mots (comme acte, agiter, agitateur, ...) ${ }^{50}$ viennent par différents cheminements du verbe latin agere. Leurs relations sont présentées dans le schéma d'agir (cf. annexe 2).

(d) $\diamond$ Les formes verbales et les adjectifs exprimant une obligation future n'ont pas une forme particulière en français mais ils en avaient une en latin et elle a laissé sa trace dans plusieurs noms français. Elle est reconnaissable à la présence du -nd- que l'on retrouve par exemple dans les mots dividende (littéralement "ce qui doit être divisé") et multiplicande ("ce qui doit être multiplié"), utilisés en mathématiques, légende ("ce qui doit être lu"), utilisé pour désigner la liste explicative des conventions de notation utilisées dans un schéma, ou offrande ("ce qui doit être offert"), utilisé pour parler d'un don fait pour respecter un rituel (religieux le plus souvent) ou une convention.

$\diamond$ Le - $a$ d'agenda est une marque flexionnelle de neutre pluriel en latin que l'on retrouve en français dans le nom masculin visa qui, comme agenda, est le produit d'une métonymie : les noms signifiant "choses à faire" ou "choses lues" en latin sont devenus les noms du registre où sont notées les choses à faire et de la marque qui est apposée sur celles qui ont été lues (des documents officiels comme des autorisations pour entrer dans certains pays).

\section{Annexe 2 : Vue partielle du schéma de la famille étymologique d'agir}

Le schéma ci-dessous ne présente que les cheminements qui séparent le verbe latin agere des mots français acte, agenda, agir, agiter et agitateur, qui ont servi à illustrer les «orientations conclusives » (cf. $\S \S 2.8,3.4$ et 4.4). D'autres mots français devront figurer dans la schématisation de la famille étymologique du verbe agir, cf. n. 50. En l'état, la représentation fournie permet d'observer que ce sont les flèches et les traits qui relient les items qui ont la charge de coder les procédés d'intégration et les relations (morphologiques et sémantiques) qui lient chaque paire de mots. Leurs formes et leurs distributions dans l'espace devraient être retravaillées pour en améliorer la lisibilité et l'esthétique, mais leur fonctionnalité est déjà posée ainsi que la répartition qui pourrait exister entre un premier niveau d'information codifiée dans la représentation graphique et des informations plus précises formulées explicitement dans les articles (comme le changement de marque flexionnelle en latin lors de la conversion d'agendus en agenda ou la modification de genre et de nombre entre ce dernier nom latin et l'emprunt français). Cette ébauche montre également que ce qui est strictement nécessaire à l'identification des mots figure dans le schéma, mais que d'autres informations ne seront mentionnées que dans les composants étymologiques associés à chaque item (catégorie et flexion, implicitement fournis dans le schéma via les gloses, localisation précise de la première attestation (texte et date en chiffres arabes), etc.). Pour présenter ce schéma à des élèves, il faudrait en outre revenir sur deux choix faits ici pour limiter la taille de l'image : éliminer les abréviations et réviser le positionnement relatif des descriptions des items anglais to agitate et agitator qui empiètent actuellement sur la ligne dévolue à l'item français agitateur. 


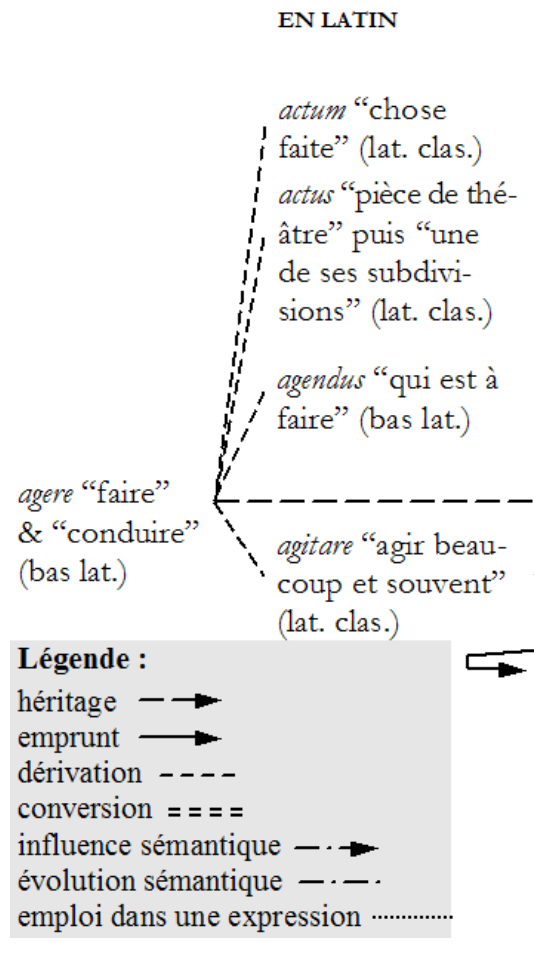

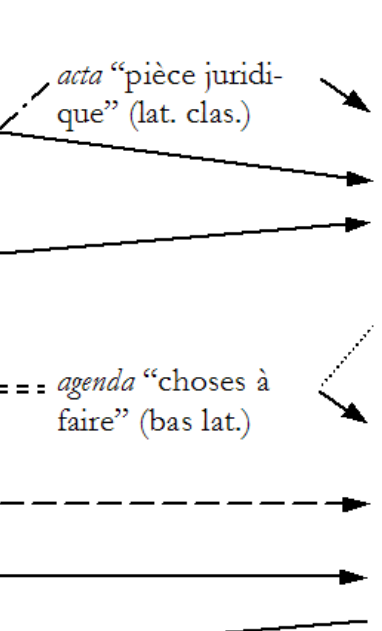

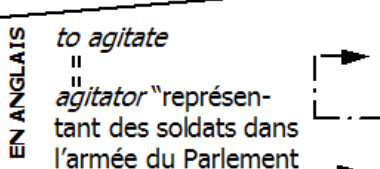

Z tant des soldats dans anglais" puis "personne qui crée ou entretien l'agitation politique ou sociale"

\section{EN FRANCAAIS}

acte

- au XIV ${ }^{e}$ s. : "pièce juridique" et plus largement "document officiel";

- au XVI ${ }^{\mathrm{e}}$ s. : "chose faite" ;

- au XVI" s. : "subdivision de pièce de théâtre" agenda diei "registre des offices du jour" (lat. ecclés. depuis le XII⿸ $\mathrm{e}$.) agenda au Xvi ${ }^{\mathrm{e}} \mathrm{s}$. "livre des comptes d'une

ville" ; depuis le XVII" $\mathrm{s}$. "registre dans lequel sont consignées les choses à faire" agir "faire" depuis le $\mathrm{Xv}^{\mathrm{e}} \mathrm{s}$. agiter

- depuis le XIII $\mathrm{e}$. : "remuer";

- depuis le XVI" s. : "perturber"; - depuis le XVII" s. s'agiter "agir beaucoup" - depuis la Révolution française : "pousser à la révolte"

agitateur depuis la Révolution française ? "personne qui crée ou entretient l'agitation politique ou sociale"

\footnotetext{
${ }^{1}$ Merci aux trois relecteurs anonymes pour leurs observations stimulantes et à Pierre Corbin pour son soutien documentaire, ses relectures critiques et ses questions "naïves" qui ont grandement contribué à ma réflexion.

${ }^{2}$ Depuis 2003, le $R J I$ intègre un « Cahier d'étymologie » $[R J I-C E]$ vendu avec le dictionnaire principal $[R J I-D]$.

${ }^{3}$ Seules les données étymologiques présentées dans les composants conçus pour les accueillir sont prises en compte dans cette contribution. Ce principe méthodologique, qui circonscrit la portée de l'étude, présente un biais puisque les dictionnaires peuvent fournir des informations de même nature dans différents composants d'articles. S.v. ouï-dire, par exemple, si le $L S M$ utilise bien le composant étymologique, dénommé " Histoire du mot», pour en mentionner l'étymologie («Vient du participe passé du verbe oü̈r et de dire : le ouï-dire est ce que l'on a entendu dire. »), elle figure en revanche dans le composant du RJI-D qui est destiné à signaler l'appartenance d'un mot à une « famille » (cf. « Avant-propos », p. VI) : « Mot de la famille de OUÏR et de DIRE. ». Cf. nn. 37 et 40 pour d'autres exemples.
}

${ }^{4}$ La présente étude s'appuie sur une base de données constituée en 2007 qui réunissait, pour une autre étude (Gasiglia 2008), les textes des composants fournissant une information étymologique d'une large sélection d'ouvrages destinés aux élèves du primaire, enrichie pour la présente circonstance de ceux du DSH et du LSM.

${ }^{5}$ Les articles de ce dictionnaire ne comportent par contre pas d'informations historiques.

${ }^{6}$ Les informations étymologiques qui ne sont pas extraites des dictionnaires scolaires mais introduites au fil de mon propos, quand elles ne sont pas référencées strictement, proviennent du Dictionnaire historique de la langue française [DHLF] et du Trésor de la langue française informatisé [TLFi].

${ }^{7}$ Le $R J I-D$ de 2003 formulait ses 165 mentions d'origines étrangères sur le patron « Ce mot vient de GLOSSONYME ». Dans le $R J I-D$ de 2005, sur les 495 mots d'origine étrangère signalés, la moitié environ des mentions sont encore écrites sur ce patron (cf. [8] et [9], § 2.6, et [e], n. 13) ou une variante de celui-ci, l'autre moitié étant enrichie.

${ }^{8}$ Quand les étymologies du $D H J$ et du $D S H$ sont textuellement identiques, la typographie reproduite est celle du $D S H$, qui, à la différence du $D H J$, affiche en gras l'item adresse en mention.

${ }^{9}$ Voir les composants étymologiques cités s.v. décapiter en [4], nucléaire en [2], ovale en [10], patience en [6] et serf en [3].

[a] s.v. fromage - « Fromage vient du latin formaticum qui signifie « qui est fait dans un moule ». » (DHJ et $D S H)$

- «Vient du latin formaticus qui signifie « moulé dans une forme, un moule ». » (LSM) 
${ }^{10}$ Voir les composants étymologiques cités s.v. armistice en [11], aube en [k] (n. 20), décapiter en [4], fromage en [a] (n. 9), nucléaire en [2] et ovale en [10].

[b] s.v. 1 bourse • «Bourse vient du latin bursa qui signifie « petit sac de cuir ». » $(D H J$ et $D S H) \bullet$ «Vient du latin bursa qui signifie « sac en cuir ». » (LSM)

[c] s.v. linoléum • «Vient du latin linum qui signifie « lin » et oleum qui signifie « huile ». » $(L S M)$

[d] s.v. plaisir • «Vient du latin placere qui signifie « plaire ». » $(L S M)$

${ }^{11}$ Les Hachette n'emploient pas « vient de » s.v. armistice, cf. [11].

${ }^{12}$ Aucun des dictionnaires étudiés ne mentionne l'anglais ; les Hachette n'emploient pas « vient de » s.v. linoléum : «Linoléum a été formé à partir de deux mots latins qui signifient «lin » et « huile », car ce matériau était enduit d'huile de lin. » (DHJ et $D S H)$.

${ }^{13}$ L'emploi de « vient de » dans le $L S M$ et le RJI-D exprime implicitement qu'il y a eu adaptation orthographique du mot anglais pour rocking-chair. Pour skate-board, le $L S M$ et le RJI ont adopté la graphie anglaise (cf. n. 14).

[e] s.v. rocking-chair - "Rocking-chair est un mot anglais formé de to rock qui signifie «balancer » et chair qui signifie « chaise ». » $(D H J$ et $D S H) \cdot$ «Vient de l'anglais to rock qui signifie «balancer » et chair qui signifie « chaise ». » $(L S M) \bullet$ « Ce mot vient de l'anglais. » $(R J I-D)$

[f] s.v. skateboard ou skate-board • «Skate-board est un mot anglais formé de to skate qui signifie « patiner » et de board qui signifie " planche ». » (DHJ et DSH, s.v. skate-board) • "Ce mot anglais vient de to skate, "patiner », et board, "planche ». » (LSM, s.v. skateboard) • «On dit aussi un skate. Ce mot vient de l'anglais skate qui veut dire « patin » et board qui veut dire « planche ». » (RJI-D, s.v. skateboard) • "C'est un mot anglais d'Amérique, composé de skate qui signifie « patin » et de board qui veut dire «planche».» (RJI-CE, s.v. skateboard)

${ }^{14}$ Graphies du Grand Robert \& Collins électronique et du Grand dictionnaire Hachette-Oxford.

${ }^{15}$ Les élèves pourraient profiter de ces explications pour mieux mémoriser les noms des éléments impliqués dans les multiplications et divisions ou de ce qui leur est fourni pour leur permettre de décoder une carte ou un schéma.

${ }^{16}$ Le $D S H$ n'indique pas qu'en latin desiderata était un nom au pluriel. Le LSM est implicite pour desiderata comme pour visa : la marque du pluriel figure dans la glose de l'étymon. Les Hachette procèdent comme le LSM pour visa (au sujet duquel le RJI ne fournit pas d'étymologie). Desiderata n'est ni à la nomenclature du $D H J$ ni à celle du $R J I$.

[g] s.v. desiderata ou désidérata • «Désidérata vient du verbe latin desiderare qui signifie « regretter l'absence de ». » (DSH, s.v. désidérata) • "C'est un mot latin qui signifie « choses désirées ». » (LSM, s.v. desiderata)

[h] s.v. visa - "Visa est un mot latin qui signifie « choses vues »: les Romains mettaient ce mot sur les actes qui avaient été vérifiés. » $(D H J$ et $D S H) \bullet$ "C'est un mot latin qui signifie « choses vues, vérifiés ». » $(L S M)$

${ }^{17}$ Voir aussi, dans le RJI-CE, s.v. serf, décapiter et patience, cf. [3], [4] et [6], et, dans le LSM, s.v. décapiter cf. [4].

${ }^{18}$ Le LSM fournit une étymologie pour chaque item (cf. [10] et [i] et [j], n. 19), mais il pourrait être plus précis encore concernant chacun et, sous une forme simplifiée appropriée, indiquer : s.v. ovale, que la dérivation d'ov(um) avec le suffixe-al(e) s'est faite en français; s.v. ovaire, qu'ov(um) a servi à dériver ovaria en latin médiéval puis ovarium en latin médical moderne, auquel le français l'a emprunté pour disposer d'ovaire (en 1672) ; s.v. ovipare, qu'ov(um) est, avec -parus ("qui met au monde"), l'un des composants d'oviparus, emprunté en 1700 pour remplacer oviperes (1518) et connu actuellement avec la forme ovipare; s.v. ovule, qu'ov(um) a également servi, avec le suffixe diminutif-ule, à former ovule, apparu en 1798.

${ }^{19}$ [i] s.v. ovaire et ovule • «Vient du latin ovum qui signifie « œuf ». » $(L S M)$

[j] s.v. ovipare • «Vient du latin ovum qui signifie « œuf » et parere qui signifie « enfanter, mettre bas ». » $(L S M)$

${ }^{20}$ Les Hachette opèrent ainsi en réorientant $s . v$. aubépine vers le texte présenté s.v. aube.

[k] s.v. aube • «Aube vient du latin alba qui signifie «blanc» et que l'on retrouve dans aubépine (épine blanche), albinos. » (DHJ et $D S H)$

[I] s.v. aubépine • « Voir aube. » (DHJ et $D S H)$

Le Dictionnaire du français vivant $[D F V]$ (déjà ancien (1971 puis 1980, cité ci-après) et destiné aux seuls collégiens) allait plus loin, en proposant des articles à nids structurés par familles étymologiques : (i) «les mots ont été groupés en familles simples. Ainsi on trouvera les deux mots ci-dessus [enfreindre et infraction] dans le même article. » (p. IV) ; (ii) «Lorsque les mots d'une grande famille sont répartis en plusieurs sous-familles, la mention du rapport étymologique est faite à chaque groupe, avec les renvois appropriés. » (p. IV). Ce principe a impliqué la mise en place d'un double système de renvois entre les mots d'une «grande famille » : (i) le composant étymologique de l'article de chaque "chef de famille" (le plus directement issu d'un étymon le plus ancien) liste les mots de sa famille décrits 
dans un autre article (dont ils constituent l'adresse principale) et à propos desquels une information étymologique est fournie (s.v. œuf : « lat. ovum. Même fam. : ove, ovaire, ovi- »); (ii) les composants étymologiques des articles de ces mots renvoient au premier (s.v. ove : « lat. ovum « œuf». V. œuf »; s.v. ovaire : « lat. scientif. ovarium; du lat. ovum «œuf ». V. œuf»; s.v. ovi-, ovo- : « du lat. ovum «œuf». V. œuf»). Les sous-articles (s.v. ovale sous ove et s.v. ovule sous ovaire) ne comportent pas d'étymologie spécifique et pas de renvoi vers le premier item (œuf).

${ }^{21}$ Comme cela a déjà été évoqué, un paratexte morphologique pourrait valoriser les procédés de construction diachroniquement stables comme la préfixation en de- en latin puis en dé- en français (cf. $\S 2.4$ à propos de décapiter). Développer ce point conduirait à sortir du périmètre thématique de la présente contribution.

${ }^{22}$ Selon le DHLF (s.v. arène), on le trouve encore "par archaïsme littéraire, jusqu'au début du XIX ${ }^{\mathrm{e}}$ s. (par ex., Chateaubriand) ».

${ }^{23}$ " Araine, areine, courants au sens de « sable » et de « grès » en ancien et moyen français (depuis 1155), viennent par voie populaire du mot latin [...] $\diamond$ La valeur latine « aire sablée (d'un cirque) », donc « lieu de combats réglés », est passée en français (arainne, XIII ${ }^{\mathrm{e}} \mathrm{s}$.). [ף] Les arènes, " amphithéâtre romain 》, est un réemprunt de la Renaissance (1539) dans la série des antiquités. $\diamond$ La valeur moderne « cirque pour les courses de taureaux », doit être un autre emprunt (1767) à l'espagnol arena, de même origine. » (DHLF, s.v. arène).

${ }^{24}$ À la différence du $D S H$, le $D H J$ présente en fait la graphie accentuée fautive aréna.

25 « emprunt, relevé par Littré (1877), à un mot anglais signifiant proprement «action de masser » (1762), substantif verbal de to shampoo « masser » (1762) puis « faire un shampooing » (1860) » (DHLF, s.v. shampooing).

${ }^{26}$ À la différence des Hachette, le LSM propose une étymologie pour shampo(o)ing, mais elle ne comporte pas d'indication sémantique, ni même catégorielle : «Vient de l'anglais shampoo. ».

${ }^{27} \mathrm{Le} D H J$ et le $R J I$ ne fournissent pas d'étymologie pour calcul.

${ }^{28}$ Dans les Hachette, s.v. acte : 1 "chose faite", 2 "document officiel" et 3 "subdivision de pièce de théâtre". Dans le $L S M$ : s.v. 1 acte, "chose faite", s.v. 2 acte, "document officiel" et s.v. 3 acte, "subdivision de pièce de théâtre". Dans le RJI-D : s.v. 1 acte, "document officiel", et s.v. 2 acte, 1 "chose faite" et 2 "subdivision de pièce de théâtre".

${ }^{29}$ Dans le RJI-CE : s.v. anorak (« au début du $20^{\mathrm{e}}$ siècle »), avion (« dans les années $\left.1920 »\right)$, biologie (« au début du $19^{\mathrm{e}}$ siècle $»$ ), budget (《à la fin du $18^{\mathrm{e}}$ siècle $)$ ), cyclone ( au milieu du $19^{\mathrm{e}}$ siècle $\left.»\right)$, dinosaure (" Au $19^{\mathrm{e}}$ siècle »), football ( «au $19^{\mathrm{e}}$ siècle »), impressionniste (« en $\left.1874 »\right)$, mammifère («à la fin du $18^{\mathrm{e}}$ siècle »), mordoré ("s'écrit à la fin du $17^{\mathrm{e}}$ siècle more doré, puis en un seul mot»), ordinateur ( au milieu du $20^{\mathrm{e}}$ siècle »), tournoi ( au $12^{\mathrm{e}}$ siècle »), verglas ( il s'écrit d'abord verreglaz au $12^{\mathrm{e}}$ siècle, puis verglas au $\left.15^{\mathrm{e}} »\right)$, zéro (« chiffre, qui apparaît au $13^{\mathrm{e}}$ siècle avec le sens de «zéro ». Il prend son sens actuel au $15^{\mathrm{e}}$ siècle, au moment où le français emprunte zéro à l'italien. »). Dans le RJI-CE, les siècles sont écrits en chiffres arabes plutôt qu'en chiffres romains.

${ }^{30}$ Selon les tables des matières d'une série de manuels scolaires actuels (Le Callennec dir.), il convient, au CE2, de permettre aux élèves de " [s]e repérer dans le temps » puis de présenter "La Préhistoire. De 4 millions d'années à 3000 avant Jésus-Christ » et «L'Antiquité. De 3000 avant Jésus-Christ à 476 après Jésus-Christ » (2007a: 4), au CM1, d'étudier «Le Moyen Âge. 476-1492» et "Des Temps modernes à la Révolution française. 1492-1815» (2007b : 4) afin de se consacrer, au CM2, aux « XIX siècle 1815-1914» et « XX $X^{\mathrm{e}}$ siècle 1914-2000» (2007c:4).

${ }^{31}$ Le latin est ensuite présenté (p. 1064) en opposant le « latin populaire » au « latin des écrivains » et en comparant les héritages que nous avons faits de mots latins sémantiquement concurrents : equus et caballus, minare et ducere.

${ }^{32}$ Un encadré présente ce texte p. 1278, dans la partie de l'ouvrage qui réunit les articles décrivant des noms propres.

${ }^{33}$ Ces événements figurent également dans la «chronologie universelle » présentée pp. 1295-1326, à l'exception des défaites des Wisigoths et des Burgondes. Villers-Cotterêts est à la nomenclature de la partie dédiée aux noms propres.

${ }^{34}$ Par exemple, le traitement en histoire, au CM1, de thèmes comme « Les grandes explorations $\left(\mathrm{XV}^{\mathrm{e}}-\mathrm{XVI}^{\mathrm{e}}\right.$ siècle) » ou "La Renaissance scientifique et technique $\left(\mathrm{XV}^{\mathrm{e}}-\mathrm{XVI}^{\mathrm{e}}\right.$ siècle) » ou encore "Les progrès techniques au XVIII siècle " (Le Callennec dir. 2007b : 4-5) peut naturellement donner lieu à des prolongements dans les autres matières d'éveil.

${ }^{35}$ Le RJI-D ne donne pas de date (« Chocolat vient de l'aztèque, qui est une langue indienne. ») et les Hachette et le $L S M$ ne fournissent pas d'étymologie. Le $L S M$, s.v. chocolat toujours mais dans le composant encyclopédique, date l'importation du chocolat en Europe et situe la diffusion de sa consommation par le peuple de France à la Révolution. 
${ }^{36}$ Le $L S M$ ne donne pas de date ( Vient du portugais banana, car ce sont les Portugais qui ont rapporté ce fruit d'Afrique. ») et les Hachette et le RJI-D ne fournissent pas d'étymologie.

${ }^{37}$ Deux langues sources évoquées mais aucune datation dans les composants étymologiques du $L S M$ ( « C'est un mot bulgare ») et du RJI-D («On dit aussi yogourt [...]. Ce mot vient du turc. ») ou dans les composants de remarques orthographiques et grammaticales du DHJ (« On dit aussi yogourt [...] qui est la forme turque de ce mot. ») et du $D S H$ (« On dit aussi yogourt ou yoghourt [...] qui est la forme turque de ce mot. »).

${ }^{38}$ Cf., respectivement : chaptaliser, galvaniser, etc. ; godillot, poubelle, etc. ; camélia, fuchsia, etc.

${ }^{39}$ [m] s.v. Pasteur • «Il découvrit que les fermentations sont dues à des organismes vivants, les microbes, dont certains provoquent des maladies infectieuses. Il élabora une technique de conservation de la bière, appelée la pasteurisation. » $(D S H)$ • «Grâce à ses expériences, Pasteur prouva que les maladies sont dues à des microbes (ou microorganismes). Entre 1870 et 1886, il découvrit le moyen d'empêcher la prolifération des micro-organismes dans les boissons (pasteurisation)» $(L S M) \bullet$ «On pasteurise le lait pour le conserver : en le chauffant et en le refroidissant brusquement, on arrête l'action destructrice des germes. » (Robert junior des noms propres $[R J N P]$ ).

${ }^{40}$ L'origine anglaise est signalée dans le composant de prononciation : «Sandwich est un terme anglais : on prononce $[\ldots] . »(D H J)$ et « Sandwich est un mot anglais : on prononce [...]. » $(D S H)$.

${ }^{41}$ Concernant ces îles, les informations finales du RJI-CE et celles du RJNP se recoupent : « [...] Le nom de ce même personnage est attaché à un archipel. En effet, l'explorateur James Cook est le premier Européen à aborder les îles Hawaii et il les baptise îles Sandwich en l'honneur de son protecteur qui état premier lord de l'Amirauté (ministre de la Marine Britannique). » (RJI-CE) et « Nom donné par James Cook à l'archipel qu'il découvre en 1778 et qui forme l'état américain d'Hawaii. » (RJNP). Le DSH et le $L S M$ ne consacrent pas d'article aux îles Sandwich.

${ }^{42}$ Le $D H J$ écrit « comte de Sandwich » en maigre.

${ }^{43}$ Le LSM (p. 1295) suggère des navettes entre sa « chronologie universelle » et ses articles : « On peut l'utiliser en la consultant à partir d'une date. [...] On peut aussi éventuellement partir d'articles du dictionnaire, dans lesquels sont mentionné[e]s des périodes ou des dates, qui permettent de se reporter à la chronologie. [...] En rebondissant ainsi du dictionnaire sur la chronologie et inversement, on peut se donner [d]es bases chronologiques et encyclopédiques ».

44 « Discours de Robespierre aux Jacobins ds BUCHEZ et ROUX, Hist. parl. Révol., XIV, 156 ds BRUNOT t. 9, p. 801 : vous n'avez sur eux d'autre avantage que d'avoir inventé le terme d' «agitateur », apparemment parce que l'autre [factieux] est usé » (TLFi, s.v. agitateur).

45 « MONTAIGNE, I, 67 ds LITTRÉ : Le dernier acte d'une comédie » (TLFi, s.v. acte $^{3}$ ).

${ }^{46}$ Les éléments fournis en annexes donnent une première idée des sélections d'informations envisagées.

${ }^{47}$ Actuellement en attente d'une nouvelle édition.

48 «Coutumes de la Salle et Chastellenie d'Ipre, 142, 3 ds Nouveau Coutumier Général, éd. Ch. A. BouRDOT DE RicheBOURG I, 848 a » (TLFi, s.v. agenda).

49 « J. LORET, La Muze hist. ou Recueil des Lettres en vers, 13 mai 1662 v. 13-15, ds BRUNOT t. 4, p. 595 » (TLFi, s.v. agenda).

${ }^{50}$ La liste dépendra de la sélection des items décrits. Dans le Dictionnaire du français vivant de 1980 (cf. n. 20), vingt items sont listés dans le composant étymologique proposé s.v. agir (« lat. agere, actus « faire, conduire, pousser à ». Même fam. : acte, actif, action, actuel, agenda, agent, agile, agiter, ambages, ambigu, essaim, essayer, exact, exaction, exiger, exigu, prodigue, réagir, rédiger, transiger »). Ces items correspondent à des adresses principales d'articles (qui comportent des composants étymologiques spécifiques et des sous-articles dont les adresses subordonnées ne sont pas mentionnées). La famille d'agir ne se limite donc pas à une vingtaine d'items puisqu'il convient de prendre en compte également les sous-adresses. Ainsi, la mention d'agenda dans la liste s.v. agir ne renvoie qu'à agenda (s.v. agenda : "mot lat. " choses à faire ». V. agir ») alors que celle d'acte renvoie à acte, acteur, trice, actuaire et entracte (s.v. acte où «lat. actum. V. agir» est précisé) et celle d'actif à actif, ive, actif (n.m.), active (n.f.s.), activement, activité, activer, s'activer, activisme, activiste, inactif, ive, inactivité, rétroactif, ive, rétroactivité, rétroactivement (s.v. actif, ive, où figure «lat. activus. V. agir»). Parmi ces sous-adresses, sept ne sont pas à la nomenclature des Hachette, du LSM, du RJI et du DMD : actuaire, actif (n.), active (n.), activisme, activiste, rétroactivité, rétroactivement. 